\title{
Absence of Single-Particle Bose-Einstein Condensation at Low Densities for Bosons with Correlated Hopping
}

\author{
Rachel Bendjama, Brijesh Kumar,* and Frédéric Mila ${ }^{\dagger}$ \\ Institute of Theoretical Physics, École Polytechnique Fédérale de Lausanne, CH 1015 Lausanne, Switzerland
}

(Received 30 June 2005; published 9 September 2005)

\begin{abstract}
Motivated by the physics of mobile triplets in frustrated quantum magnets, the properties of a twodimensional model of bosons with correlated hopping are investigated. A mean-field analysis reveals the presence of a pairing phase without single-particle Bose-Einstein condensation (BEC) at low densities for sufficiently strong correlated hopping, and of an Ising quantum phase transition towards a BEC phase at larger density. The physical arguments supporting the mean-field results and their implications for bosonic and quantum spin systems are discussed.
\end{abstract}

DOI: 10.1103/PhysRevLett.95.110406

PACS numbers: 05.30.Jp, 67.40.Db, 75.10.Jm

The models of interacting bosons (with or without disorder) have been a subject of active research. They are studied for a variety of reasons, coming from different experimental systems, such as Josephson junction arrays [1], ${ }^{4} \mathrm{He}$ in porous media [2], disordered films with superconducting and insulating phases [3], or more recently in the context of atoms trapped on an optical lattice [4]. The interplay of interaction, disorder, and kinetic energy leads to the ground states that can be a superfluid, a Bose glass, a Mott insulator, or a supersolid [5-11]. In the context of spin models too, the Schwinger boson mean-field theories provide a useful description of magnetism in the bosonic language [12-15].

Over the past decade, bosons have also been used in the context of quantum magnetism to describe the magnetization process of gapped systems with a singlet ground state such as spin ladders, the triplets induced by the magnetic field being treated as hard-core bosons. These bosons may condense, leading to the ordering of the transverse component of the spins, but they might as well undergo a superfluid-insulator transition, leading to magnetization plateaux [16]. For pure SU(2) interactions, and without disorder, the common belief is that the only alternative, not realized so far in quantum magnets, is a supersolid, i.e., a coexistence of these phases.

In this Letter, we propose that there is another possibility, namely, a pairing phase without single-particle Bose condensation. Our starting point is the observation that the effective bosonic model of a frustrated quantum magnet such as $\mathrm{SrCu}_{2}\left(\mathrm{BO}_{3}\right)_{2}$ [17] contains, in addition to the usual kinetic and potential terms, a correlated hopping term where a boson can hop only if there is another boson nearby, and that this term can be the dominant source of kinetic energy in geometries such as the orthogonal dimer model realized in $\mathrm{SrCu}_{2}\left(\mathrm{BO}_{3}\right)_{2}$ [18,19]. While the possibility of bound state formation was already pointed out in that context, the consequences of the presence of such a term on the phase diagram at finite densities have not been worked out yet.
For clarity, we concentrate in this Letter on a minimal version of the model, but we have checked that the conclusions apply to the more realistic model derived for $\mathrm{SrCu}_{2}\left(\mathrm{BO}_{3}\right)_{2}$ [20]. This model is defined on a square lattice by the Hamiltonian

$$
\begin{aligned}
H= & -t \sum_{\mathbf{r}} \sum_{\delta= \pm x, \pm y} b_{\mathbf{r}+\delta}^{\dagger} b_{\mathbf{r}}-\mu \sum_{\mathbf{r}} n_{\mathbf{r}} \\
& -t^{\prime} \sum_{\mathbf{r}} \sum_{\delta= \pm x} \sum_{\delta^{\prime}= \pm y} n_{\mathbf{r}}\left\{b_{\mathbf{r}+\delta}^{\dagger} b_{\mathbf{r}+\delta^{\prime}}+\text { H.c. }\right\},
\end{aligned}
$$

where $b_{\mathbf{r}}^{\dagger}$ and $b_{\mathbf{r}}$ are boson operators and $n_{\mathbf{r}}=b_{\mathbf{r}}^{\dagger} b_{\mathbf{r}}$. $t$ and $t^{\prime}$ are the measures of single-particle and correlated hopping, respectively (see Fig. 1). A hard-core constraint that excludes multiple occupancy should in principle be included. However, we concentrate on the low density limit, where this constraint is expected to be irrelevant. So in the following we work with regular (soft-core) bosons.

Since the correlated hopping term in Eq. (1) is quartic in the single-particle boson operators, the simplest thing to do is a mean-field theory. Since the system gains energy through correlated hopping by having two particles nearby, a natural choice for a mean field is the pairing amplitude. The particle density and the kinetic amplitudes are the other choices for the mean fields. In the following, we formulate a mean-field theory in terms of these order

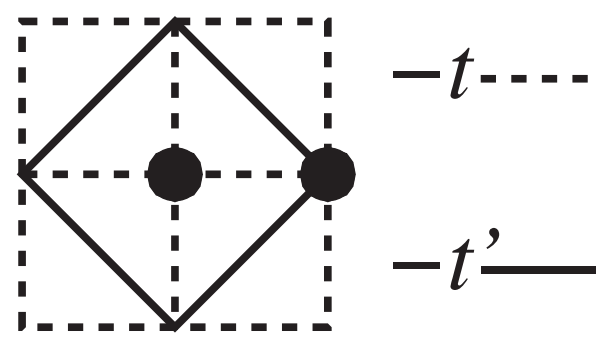

FIG. 1. The diagonal bonds $\left(-t^{\prime}\right)$ denote a correlated hopping process, and the nearest neighboring bonds denote the singleparticle hopping $(-t)$. 
parameters defined by

$$
\begin{aligned}
\Delta & =\left\langle b_{\mathbf{r}}^{\dagger} b_{\mathbf{r} \pm \delta}^{\dagger}\right\rangle \quad \text { (pairing amplitude) } \\
\kappa & =\left\langle b_{\mathbf{r}}^{\dagger} b_{\mathbf{r} \pm \delta}\right\rangle, \\
\kappa^{\prime} & =\left\langle b_{\mathbf{r} \pm \delta}^{\dagger} b_{\mathbf{r} \pm \delta^{\prime}}\right\rangle \quad \text { (kinetic amplitudes), } \\
n & =\left\langle b_{\mathbf{r}}^{\dagger} b_{\mathbf{r}}\right\rangle \quad \text { (particle density), }
\end{aligned}
$$

where $\delta \neq \delta^{\prime}$, and $\delta, \delta^{\prime}=x, y$. The particle density is taken to be uniform and the kinetic amplitudes real. In principle, we can allow for an internal phase in the pairing amplitude (a nonzero phase between $x$ and $y$ direction bonds) as in the mean-field theory of the $t$ - $J$ model in the context of the high- $T_{c}$ cuprates. Here, we take the internal phase to be zero (the extended $s$-wave pairing). The corresponding mean-field Hamiltonian has the following form:

$$
H_{\mathrm{MF}}=\mathcal{E}_{0}+\sum_{\mathbf{k}}\left\{\xi_{\mathbf{k}} b_{\mathbf{k}}^{\dagger} b_{\mathbf{k}}-\Delta_{\mathbf{k}}\left[b_{\mathbf{k}}^{\dagger} b_{-\mathbf{k}}^{\dagger}+\text { H.c. }\right]\right\},
$$

where $\mathcal{E}_{0}, \xi_{\mathbf{k}}$, and $\Delta_{\mathbf{k}}$ are given by

$$
\begin{aligned}
\mathcal{E}_{0}= & 8 t^{\prime} L\left[\Delta^{2}+\kappa^{2}+n \kappa^{\prime}\right], \\
\xi_{\mathbf{k}}= & -2\left(t+4 t^{\prime} \kappa\right)\left(\cos k_{x}+\cos k_{y}\right)-8 t^{\prime} n \cos k_{x} \cos k_{y} \\
& -\left(\mu+8 t^{\prime} \kappa^{\prime}\right) \\
\Delta_{\mathbf{k}}= & 4 t^{\prime} \Delta\left(\cos k_{x}+\cos k_{y}\right) .
\end{aligned}
$$

The Hamiltonian $H_{\mathrm{MF}}$ can easily be diagonalized using Bogoliubov transformation for bosons. The canonical free energy density for $H_{\mathrm{MF}}$ is given as

$$
\begin{aligned}
f= & \lambda\left(n+\frac{1}{2}\right)+8 t^{\prime}\left(\Delta^{2}+\kappa^{2}\right)+\frac{1}{2 L} \sum_{\mathbf{k}} E_{\mathbf{k}} \\
& +\frac{1}{\beta L} \sum_{\mathbf{k}} \log \left(1-e^{-\beta E_{\mathbf{k}}}\right),
\end{aligned}
$$

where $E_{\mathbf{k}}=\sqrt{\xi_{\mathbf{k}}^{2}-4 \Delta_{\mathbf{k}}^{2}}$ is the quasiparticle dispersion, $\beta=1 / k_{B} T$, and $\lambda=\mu+8 t^{\prime} \kappa^{\prime}$ is the effective chemical potential. Redefining the chemical potential in this way makes $\kappa^{\prime}$ a redundant order parameter in the mean-field theory. Note that $\mu$ and $\kappa^{\prime}$ appear with the right combination to give $\lambda$ as the new chemical potential in $\xi_{\mathbf{k}}$. Hence, for a given $n, f$ is purely a function of $\lambda, \kappa$, and $\Delta$.

The self-consistent equations for the order parameters can be written

$$
\begin{gathered}
n=n_{c}-\frac{1}{2}+\frac{1}{2 L} \sum_{\mathbf{k}} \frac{\xi_{\mathbf{k}}}{E_{\mathbf{k}}} \operatorname{coth} \frac{\beta E_{\mathbf{k}}}{2} \\
\kappa=n_{c}+\frac{1}{4 L} \sum_{\mathbf{k}} \frac{\xi_{\mathbf{k}}\left(\cos k_{x}+\cos k_{y}\right)}{E_{\mathbf{k}}} \operatorname{coth} \frac{\beta E_{\mathbf{k}}}{2}, \\
\Delta=n_{c}+\frac{2 t^{\prime} \Delta}{L} \sum_{\mathbf{k}} \frac{\left(\cos k_{x}+\cos k_{y}\right)^{2}}{E_{\mathbf{k}}} \operatorname{coth} \frac{\beta E_{\mathbf{k}}}{2},
\end{gathered}
$$

where $n_{c}$ is the condensate density (the occupancy of the zero-energy mode if any). Since the model is twodimensional, $n_{c}=0$ for $T>0$. At $T=0, n_{c}$ may or may not be zero, and solutions must be searched with two strategies: assume $n_{c}=0$ and solve these equations for the unknowns $(\kappa, \Delta, \lambda)$, or assume there is a zero-energy mode (which fixes $\lambda$ ) and solve for the unknowns $(\kappa, \Delta$, $\left.n_{c}\right)$. In that case, the wave vector corresponding to the zeroenergy mode ( $\mathbf{k}=0$ here) must be excluded from the sum. If several solutions are found for a given density, the one with the lowest energy should be chosen. In practice, we found only one solution for a given density. In general, these equations are solved by simple iteration. Note, however, that, when $n_{c}=0$, Eqs. (4) and (5) can still be solved by iteration, but Eq. (6) needs to be solved for $\Delta$ by some numerical method at each step of the iteration.

Let us first discuss the $T=0$ results. The most remarkable feature is that it turned out to be impossible to find a solution with a nonzero condensate at low enough density unless $t^{\prime}$ is very small. In other words, as soon as the correlated hopping is not too small, there is no singleparticle Bose-Einstein condensation (BEC) at low density. The critical density $n^{*}$ below which this is the case is plotted as a function of $\alpha=t^{\prime} /\left(t+t^{\prime}\right)$ in Fig. 2. This figure calls for some comments. First of all, $t^{\prime} / t$ need not be large for the effect to be observable, which ensures the relevance of the present discussion for the quantum magnets such as $\mathrm{SrCu}_{2}\left(\mathrm{BO}_{3}\right)_{2}$. Besides, the critical value $n^{*}$ is quite small even when $t^{\prime}$ dominates, and our approximation to treat triplets as soft-core bosons is expected to be good in the whole range of Fig. 2. Finally, densities of a few percent are definitely accessible in the context of quantum magnets, the density being equivalent to the magnetization relative to the saturation value.

Next we turn to the nature of this non-BEC phase. Clearly this cannot be a commensurate insulating phase of the type observed before since it occurs for a range of densities. In fact, its nature is best revealed by looking at the order parameters. While $n_{c}=0$ in the pairing phase

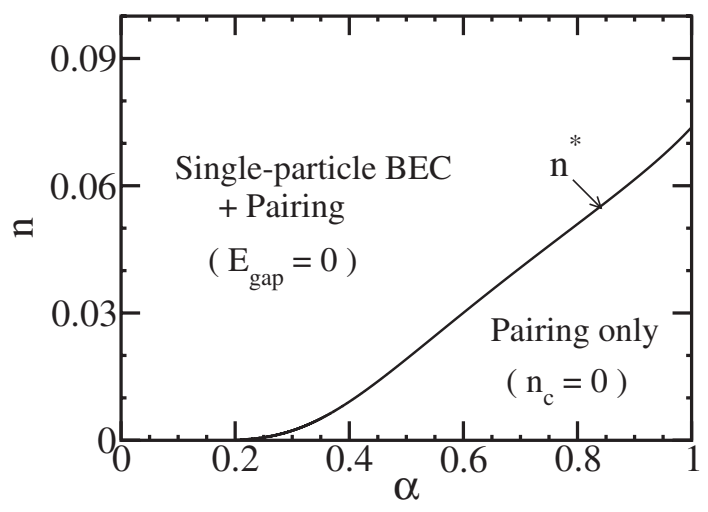

FIG. 2. The mean-field quantum phase diagram. $n^{*}$ is the critical density at which, for a given $\alpha=t^{\prime} /\left(t+t^{\prime}\right)$, the singleparticle condensate density $n_{c}$ becomes zero. This marks the onset of the pairing phase with gapped quasiparticle excitations. 


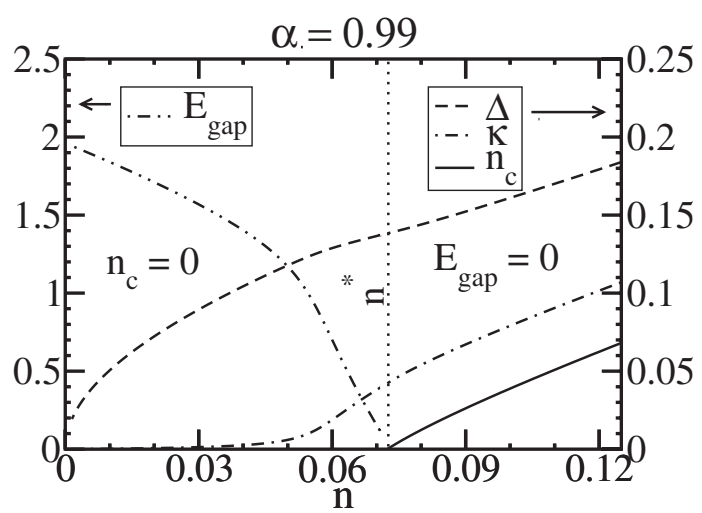

FIG. 3. The variation of the quasiparticle gap and the meanfield order parameters as a function of $n$ for a fixed $\alpha$. While $n_{c} \neq 0$ only in the single-particle BEC phase $(n \gtrsim 0.072)$, the pairing amplitude $\Delta \neq 0$ in both phases.

and $E_{\text {gap }}=0$ in the single-particle BEC phase, the meanfield solution for $\kappa$ and $\Delta$ is nonzero on both sides. In Fig. 3 the behavior of $n_{c}, \kappa$, and $\Delta$ is shown as a function of $n$ for $\alpha=0.99$. It is not surprising that $\Delta$ is nonzero in the single-particle BEC phase. In fact, the single-particle BEC state means $\left\langle b^{\dagger}\right\rangle \neq 0$, which further implies that $\left\langle b^{\dagger} b^{\dagger}\right\rangle \approx\left\langle b^{\dagger}\right\rangle^{2} \neq 0$. Hence $\Delta$ will always be nonzero in the single-particle BEC phase. The correct measure of the existence of the pairing (independent of the contribution from the single-particle BEC) is $\Delta-n_{c}$. We know from the calculation (see Fig. 4) that for the noninteracting Bose gas $(\alpha=0), \Delta=n_{c}$, as it should be. However, for any finite $\alpha$ we find $\Delta-n_{c}>0$. Thus, for arbitrarily small values of the correlated hopping, the system develops a tendency towards pair formation. However, it does not suppress the single-particle BEC in favor of a purely pairing phase until sufficiently strong $\alpha$ is reached for sufficiently small $n$.

The results of our mean-field calculation are similar to those obtained on a different problem in the context of the atomic gases [21]. These are studies regarding the transition from a purely molecular condensate (MC) to an atomic condensate $(\mathrm{AC})$ with a nonzero fraction of the molecular condensate present (AC $+\mathrm{MC})$ across the Feshbach resonance. Our pairing phase is like the $\mathrm{MC}$, and the singleparticle $\mathrm{BEC}$ phase is analogous to the $\mathrm{AC}+\mathrm{MC}$.

The nature of the transition based on symmetry considerations is also similar. The mean-field Hamiltonian, $H_{\mathrm{MF}}$, explicitly breaks the U(1) gauge symmetry; however, it is still invariant under global $Z_{2}$ symmetry, that is, under $b_{\mathbf{r}} \rightarrow-b_{\mathbf{r}}$. In other words, gauge symmetry, $b_{\mathbf{r}}^{\dagger} \rightarrow b_{\mathbf{r}}^{\dagger} e^{i \phi}$ leaves $H_{\mathrm{MF}}$ invariant for $\phi=\pi$. This residual Ising-like symmetry will also be broken if there is single-particle BEC (because $\langle b\rangle \neq 0$ ). What we have in Fig. 2 is such an Ising symmetry breaking quantum phase transition, where $n_{c}$ is the relevant order parameter. The pairing phase respects this $Z_{2}$ symmetry while the single-particle BEC phase breaks it spontaneously.

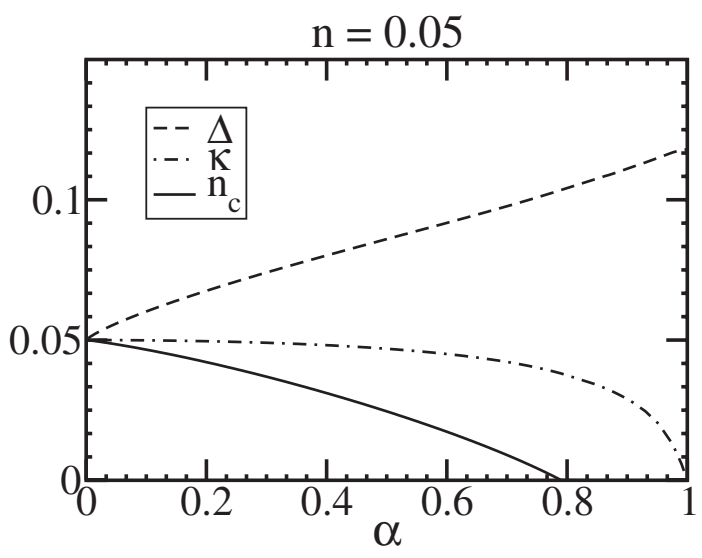

FIG. 4. The behavior of different order parameters as a function of $\alpha$ for $n=0.05$. For $\alpha \gtrsim 0.78$, the condensate density $\left(n_{c}\right)$ vanishes, and the system goes into the pairing ground state.

The temperature dependence of various quantities in the mean-field theory is shown in Fig. 5. The temperature at which $\Delta$ becomes zero is called $T_{c}$. This quantifies the mean-field phase transition from a normal Bose gas at high temperatures to a pairing phase below $T_{c}$. The inset of Fig. 5 shows $T_{c}$ as a function of $n$. Remarkably, there is no detectable anomaly upon going through the critical density $n^{*}$. Since we are in 2D, we do not expect to have a true BEC of pairs, but rather a Kosterlitz-Thouless (KT) transition. These results suggest that the system should undergo one KT transition whatever the density, followed by an Ising transition if $n>n^{*}$.

To check the validity of the mean-field approximation, hence of our conclusions, it would be very useful to have unbiased numerical results on the model of Eq. (1). However, we have good reasons to believe that the predictions of the present mean-field theory are physically relevant. Mathematically, the structure of the mean-field equations and the results of the calculations are similar to the Schwinger boson mean-field theory of the quantum spin system $[13,15]$. In that context, the single-particle BEC phase implies an ordered phase in the spin variables, while the pairing phase denotes a disordered phase. Now the physical relevance of these disordered phases is well established in the context of quantum magnets [22,23], and we expect the same to be true here.

The model studied in this Letter has similarities with the ring exchange model of bosonic Cooper pairs introduced some time ago by Paramekanti and collaborators [24]. In their model, the pairs of bosons hop on the opposite corners of a plaquette. The low temperature physics is significantly different however. In their model, the number of bosons is conserved on each row and column of the square lattice, leading to the Luttinger liquid like physics and critical correlations in the ground state. In our model, the correlated hopping does not sustain any such conservation law, and the ground state is expected to develop a true longrange order. 


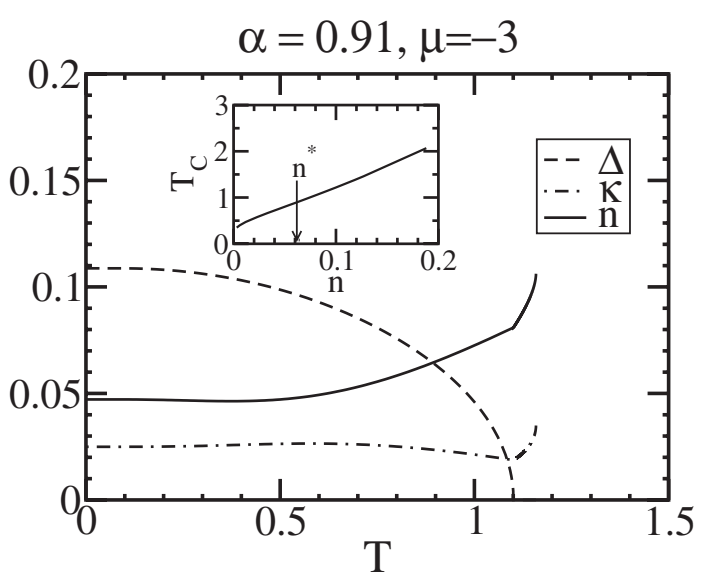

FIG. 5. The temperature dependence of $\Delta, \kappa$, and $n$ for a given $\mu$ and $\alpha$. Inset: dependence of $T_{c}$ on the density.

Finally, let us briefly discuss the physical implications of these results for the magnetization process of gapped quantum magnets. The thermodynamics was already discussed in the boson language: we expect to observe a KT transition for any magnetization, followed by an Ising transition if the magnetization is larger than a critical value. This will remain essentially true for 3D systems, the KT transition being replaced by a true phase transition toward an ordered phase. However, we also expect very significant differences between the zero temperature phases. Single-particle BEC means magnetic long-range order, and the system is expected to have gapless transverse spin waves. In other words, the gap detected in spectroscopies such as inelastic neutron scattering or NMR will vanish. However, at low magnetization, we have only pair BEC. The order implied by this pair BEC will be of nematic type since the transverse components of the spins within a pair can be flipped without changing the correlations. But more importantly, there is a gap to single-particle excitations, i.e., to single spin flips. Although the system is gapless in this phase, we thus expect to observe a gap in neutron scattering or NMR, the gapless excitations appearing only in the channel $\Delta S=2$.

In summary, we have shown that the correlated hopping can change drastically the properties of bosons, leading at low densities to a pairing phase without single-particle BEC, and with gapped quasiparticle excitations. In the context of quantum frustrated magnets, this leads to the prediction of an Ising phase transition (for low magnetization) as a function of the magnetic field, for systems where frustration reduces direct hopping of triplets, thus making correlated hopping the dominant process of kinetic energy.

Beyond frustrated magnets, these results will have implications on all systems where correlated hopping may be the dominant source of kinetic energy. One such class of systems is the atomic gases, where different external parameters control the hopping and the Coulomb terms. Whether instabilities of the kind described here can be induced in these systems by reducing the single-particle kinetic energy is left for future investigation.

We thank A. Georges, S. Miyahara, D. Poilblanc, and M. Troyer for stimulating discussions on different aspects of this work. We also acknowledge the Swiss National Funds and the MaNEP for the financial support.

*Electronic address: brijesh.kumar@epfl.ch

†Electronic address: frederic.mila@epfl.ch

[1] L. J. Geerligs, M. Peters, L. E. M. de Groot, A. Verbruggen, and J. E. Mooij, Phys. Rev. Lett. 63, 326 (1989).

[2] M. H. W. Chan, K. I. Blum, S. Q. Murphy, G. K. S. Wong, and J. D. Reppy, Phys. Rev. Lett. 61, 1950 (1988).

[3] D. B. Haviland, Y. Liu, and A. M. Goldman, Phys. Rev. Lett. 62, 2180 (1989).

[4] M. Greiner, O. Mandel, T. Esslinger, T. W. Hänsch, and I. Bloch, Nature (London) 415, 39 (2002).

[5] M. P. A. Fisher, P. B. Weichman, G. Grinstein, and D. S. Fisher, Phys. Rev. B 40, 546 (1989).

[6] T. Klien, I. Joumard, S. Blanchard, J. Marcus, R. Cubitt, T. Giamarchi, and P.L. Doussal, Nature (London) 413, 404 (2001).

[7] F. Alet and E. S. Sørensen, Phys. Rev. B 70, 024513 (2004).

[8] J. K. Freericks and H. Monien, Phys. Rev. B 53, 2691 (1996).

[9] K. Sheshadri, H. R. Krishnamurthy, R. Pandit, and T. V. Ramakrishnan, Phys. Rev. Lett. 75, 4075 (1995).

[10] P. Sengupta, L. P. Pryadko, F. Alet, M. Troyer, and G. Schmid, Phys. Rev. Lett. 94, 207202 (2005).

[11] A. van Otterlo and K.-H. Wagenblast, Phys. Rev. Lett. 72, 3598 (1994).

[12] D. P. Arovas and A. Auerbach, Phys. Rev. B 38, 316 (1988).

[13] S. Sarkar, C. Jayaprakash, H. R. Krishnamurthy, and M. Ma, Phys. Rev. B 40, 5028 (1989).

[14] P. Chandra, P. Coleman, and A. I. Larkin, J. Phys. Condens. Matter 2, 7933 (1990).

[15] F. Mila, D. Poilblanc, and C. Bruder, Phys. Rev. B 43, 7891 (1991).

[16] T. M. Rice, Science 298, 760 (2002), and see references therein.

[17] H. Kageyama, K. Yoshimura, R. Stern, N. V. Mushnikov, K. Onizuka, M. Kato, K. Kosuge, C. P. Slichter, T. Goto, and Y. Ueda, Phys. Rev. Lett. 82, 3168 (1999).

[18] T. Momoi and K. Totsuka, Phys. Rev. B 62, 15067 (2000).

[19] S. Miyahara and K. Ueda, J. Phys. Condens. Matter 15, R327 (2003).

[20] R. Bendjama, B. Kumar, and F. Mila (to be published).

[21] M. W. H. Romans, R. A. Duine, S. Sachdev, and H. T. C. Stoof, Phys. Rev. Lett. 93, 020405 (2004).

[22] S. Chakravarty, B. I. Halperin, and D. Nelson, Phys. Rev. B 39, 2344 (1989).

[23] S. Sachdev, Quantum Phase Transitions (Cambridge University Press, Cambridge, U.K., 1999).

[24] A. Paramekanti, L. Balents, and M. P. A. Fisher, Phys. Rev. B 66, 054526 (2002). 